OPEN ACCESS

Edited by: Yong Wang,

Zhejiang University, China

Reviewed by:

Hongbin Wan,

Novartis Institutes for BioMedical

Research, United States

Chao-Yie Yang,

University of Tennessee Health

Science Center (UTHSC),

United States

Hao-Jen Hsu,

Tzu Chi University, Taiwan

*Correspondence:

George P. Lisi

george_lisi@brown.edu

Specialty section:

This article was submitted to

Biophysics,

a section of the journal

Frontiers in Molecular Biosciences

Received: 09 September 2021

Accepted: 06 October 2021

Published: 25 October 2021

Citation:

Cui JY and Lisi GP (2021) Molecular Level Insights Into the Structural and

Dynamic Factors Driving

Cytokine Function.

Front. Mol. Biosci. 8:773252.

doi: $10.3389 /$ /fmolb.2021.773252

\section{Molecular Level Insights Into the Structural and Dynamic Factors Driving Cytokine Function}

\author{
Jennifer Y. Cui and George P. Lisi * \\ Department of Molecular Biology, Cell Biology and Biochemistry, Brown University, Providence, RI, United States
}

Cytokines are key mediators of cellular communication and regulators of biological advents. The timing, quantity and localization of cytokines are key features in producing specific biological outcomes, and thus have been thoroughly studied and reviewed while continuing to be a focus of the cytokine biology community. Due to the complexity of cellular signaling and multitude of factors that can affect signaling outcomes, systemic level studies of cytokines are ongoing. Despite their small size, cytokines can exhibit structurally promiscuous and dynamic behavior that plays an equally important role in biological activity. In this review using case studies, we highlight the recent insight gained from observing cytokines through a molecular lens and how this may complement a system-level understanding of cytokine biology, explain diversity of downstream signaling events, and inform therapeutic and experimental development.

Keywords: cytokine, protein dynamics, immunology, spectroscopy, allostery

\section{INTRODUCTION}

Cytokines are small signaling proteins that serve as effectors of immunity and require spatial or temporal expression to regulate biological outcomes (Altan-Bonnet and Mukherjee, 2019). Cytokines are especially prevalent in disease states where their presence can indicate pathological progression or conversely, a protective effect. For example, signaling cascades that maintain homeostatic balance use cytokines to coordinate appropriate responses that initiate or terminate inflammation. The lens through which researchers often view cytokine biochemistry involves their localization and quantity, using phenomenological models to derive system-level explanations of function (Altan-Bonnet and Mukherjee, 2019). Despite the utility of this approach, the molecular aspects of cytokine function remain unclear, hindering efforts to intuitively modulate their mechanisms and promiscuous interactions. Understanding the structural and environmental factors that stimulate transformations of cytokines is critical to disentangling their role in biological events. Such insight can inform 1) the role played by cytokines as agents of cellular communication, 2) the improvement of tools leveraged for research, and 3) the development of cytokines as therapeutics or targets in clinical treatment plans. In the past decade, many enzymes and metabolic proteins that accommodate multiple non-overlapping functions by "moonlighting" have been shown to utilize intrinsic protein dynamics as a mechanistic driving force (Mani et al., 2015; Boukouris et al., 2016; Chen et al., 2018; Chen et al., 2021). However, structural plasticity in cytokines with broadly demonstrated abilities to harbor multiple functions within compact scaffolds has not been as thoroughly investigated as a moonlighting mechanism.

Researchers use cytokines to replicate immunological conditions in the laboratory in order to study behaviors of specific cell types (Schluns and Lefrançois, 2003; Arango Duque and Descoteaux, 
2014; Roan et al., 2019), to replicate extracellular environments, to observe biological responses in vitro (Kämpfer et al., 2017; Berghaus et al., 2018; Barnes and Somerville, 2020), and as a measurable biomarker of disease. Cytokines are also used as therapeutics in clinical settings to specifically stimulate immune responses, including interleukins (ILs) in cancer and colony-stimulating factors (CSFs) in general neutropenia (Mehta et al., 2015; Berraondo et al., 2019; Conlon et al., 2019). Given the breadth of the roles played by cytokines intrinsically and as research tools or in clinical settings (i.e. from bench to bedside), it is clear that having thorough understanding of the mechanisms driving cytokine function is crucial. One factor that complicates structure-function investigations of cytokines, and the establishment of general rules that govern their function, is the fact that these proteins exert a great deal of their influence under conditions of disease, which are more difficult to mimic in structural characterizations. In this Review, we discuss current efforts to define the mechanisms of cytokines at the "residue level," where changes in atomic structure or conformational dynamics of the protein yields information relevant to physiological scenarios. We highlight case studies of cytokines with emerging diverse roles, while also showcasing experimental and theoretical approaches for understanding cytokine biochemistry. Though we cannot comprehensively discuss every avenue of investigation in this Review (Künze et al., 2014; Herring et al., 2015; Joseph et al., 2015; Künze et al., 2016; Park et al., 2017; Azimzadeh Irani and Ejtehadi, 2019; Penk et al., 2019; Gangele et al., 2020; Roy, 2020; Yang, 2020), each of the case studies below offers a useful piece insight into an extremely complex class of biomolecules that may aid in the development of broadly applicable strategies to study molecular interactions.

\section{CASE STUDY I: MACROPHAGE MIGRATION INHIBITORY FACTOR}

Macrophage migration inhibitory factor (MIF) is implicated in a wide range of disease states, where elevated MIF levels are markers for tumorigenesis (Bach et al., 2008; Noe and Mitchell, 2020), sepsis (Toldi et al., 2021), acute respiratory distress syndrome (ARDS) (Donnelly et al., 1997; Lai et al., 2003), arthritis (Onodera et al., 1999; Kim et al., 2007; LlamasCovarrubias et al., 2012), colitis (de Jong et al., 2001), and Malaria (Han et al., 2010; Sun et al., 2012; Baeza Garcia et al., 2018). MIF is highly conserved in diverse organisms and is heavily involved in inflammatory responses, cell cycle control, the sensing of pathogenic stimuli, activation of innate defense responses, recruitment of immune cells, and prevention of p53-mediated apoptosis (Hudson et al., 1999; Mitchell et al., 2002). Despite a compact structural scaffold and a lack of clear modular domains, MIF contains several sequence motifs, including two enzymatic sites for disparate tautomerase and oxidoreductase activities (a third nuclease activity was recently proposed), that provide MIF with a surprising functional complexity (Kleemann et al., 1998; Lubetsky et al., 1999; Wang et al., 2021). MIF is also remarkably amenable to interactions with a number of target proteins, despite its overall architectural rigidity (Mühlhahn et al., 1996), and is proposed to be involved, directly or indirectly, in 49 signaling events. (Subbannayya et al., 2016). This functional promiscuity necessitates an intimate understanding of the mechanism(s) that govern its cellular interactions, but also complicate efforts to assign any singular function to MIF with a high level of mechanistic detail.

Efforts to understand MIF at the molecular level have focused on 1) studies of mutations that affect its structure and function, 2) studies of signaling within its homotrimeric structure, and 3) studies of interactions between MIF and drug-like molecules (Lubetsky et al., 1999; Mühlhahn et al., 1996; Pantouris et al., 2015; Pantouris et al., 2020; Fan et al., 2013; Trivedi-Parmar and Jorgensen, 2018; Cournia et al., 2009; Cho et al., 2010; Takahashi et al., 2009). Site-directed mutagenesis has been employed by several laboratories to examine the structural and biological impact of perturbations to the MIF catalytic and receptor binding sites (Pantouris et al., 2015; Pantouris et al., 2020; Fan et al., 2013; El-Turk et al., 2012; Oda et al., 2008; El-Turk et al., 2008; Bendrat et al., 1997). X-ray crystallographic reports of $>100$ MIF structures in the Protein Data Bank show essentially no observable changes in response to mutations, substrates or inhibitors, suggesting MIF is highly rigid and resistant to architectural deformation. However, identical solution NMR experiments show that atomic level fingerprints of MIF variants are unique, indicating that the local structure of MIF is in fact quite flexible in solution and may play a significant role in modulating its biochemistry (Pantouris et al., 2018; Schinagl et al., 2018). More recent work has leveraged these findings to explore the existence of signaling pathways that propagate chemical information between distant regions of the MIF structure. Pantouris, et al characterized two such pathways in successive reports, first demonstrating through molecular dynamics (MD) simulations that nanosecond timescale conformational fluctuations were critical to the organization of the binding interface for the MIF-induced activation of CD74 (Pantouris et al., 2015). Further analysis of correlated dynamics in MIF revealed the CD74 activation signal to originate from a previously uncharacterized residue, Tyr99, at the solvent channel formed by the three-fold symmetric MIF structure (Figure 1A). Interestingly, this allosteric site modulates the dynamics of the MIF protein on multiple timescales when mutated, and not only affects CD74 activation in vivo, but also regulates tautomerase enzymatic activity (Figure 1B) through a different signaling pathway originating from Tyr99 (Pantouris et al., 2018; Pantouris et al., 2020; Parkins et al., 2021). The factors that influence the ramping up or down of MIF enzymatic activity include protein dynamics, intramolecular hydrogen bonds and hydrogen bonds with solvent molecules, and pi-stacking interactions from aromatic side chains lining the signaling pathway. At present, it is still unclear how, or if, CD74 activation or tautomerase activity are preferentially activated in the cell. Nonetheless, recent solution studies of MIF revealed two of its biochemical functions to be connected by a region of its structure previously uncharacterized at the molecular level. Numerous studies of MIF in complex with drugs and other modulators have never targeted the Tyr99 regulatory site, but 

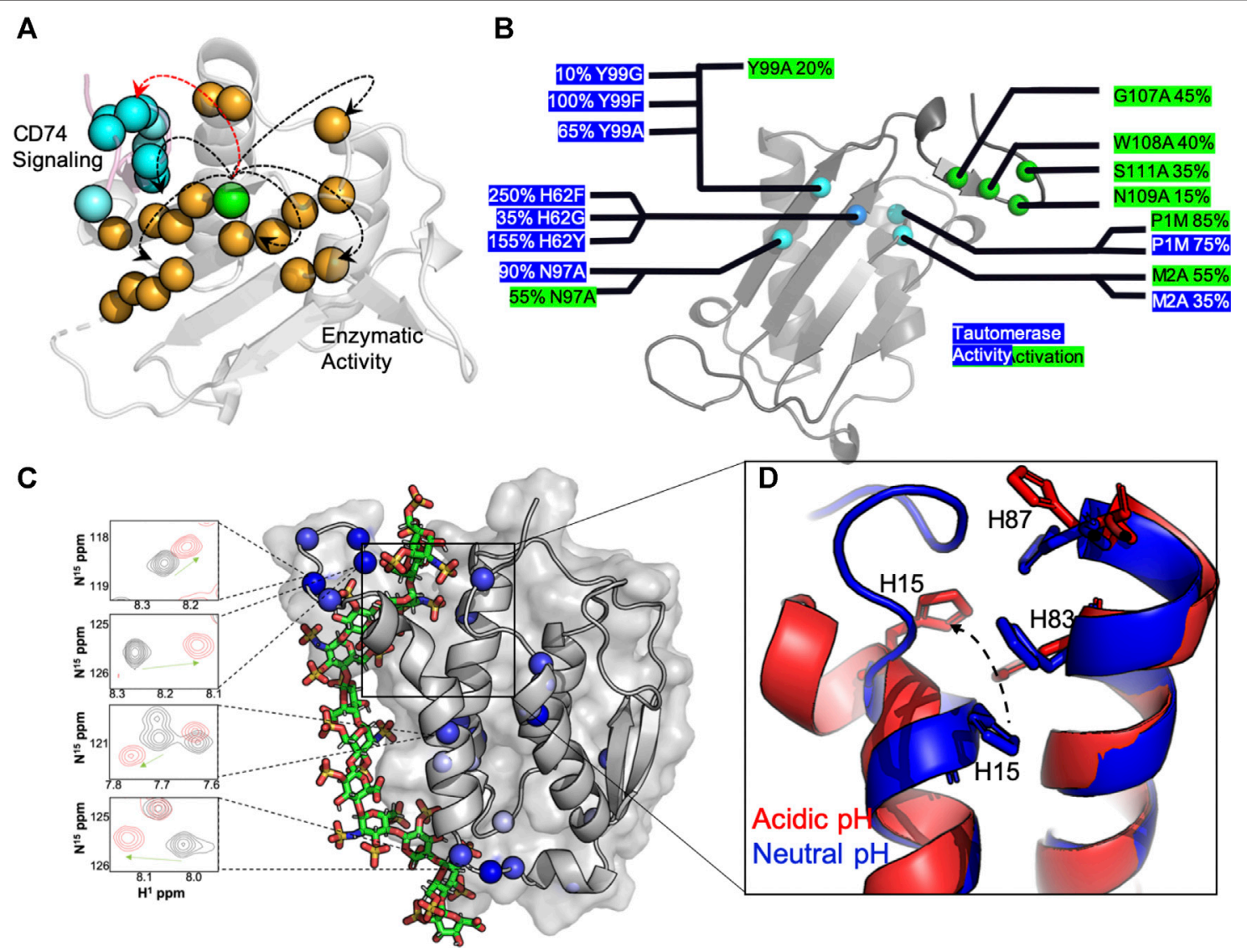

FIGURE 1 | Molecular insights from structure-function studies of MIF and GMCSF. (A) Tyr99 mutations (green) induce widespread NMR chemical shifts and line broadening (orange spheres), suggesting that are large portion of the MIF structure "senses" short and long-range correlations (arrows) that influence MIF signaling. The C-terminus of the adjacent MIF monomer, the site of CD74 activation, is shown in pink with blue spheres. (B) Experimental measurements of MIF activities determined from mutagenesis. Activity levels are presented as a percentage of wild-type (wt) MIF activity, with enzymatic catalysis (blue) determined from the enol-keto tautomerization of 4-hydroxyphenyl pyruvate (4-HPP) (Lubetsky et al., 1999) and CD74 activation (green) determined from the recruitment of neutrophils to murine lungs in vivo (Takahashi et al., 2009). (C) A computationally-derived GMCSF structure in the presence of a heparin oligo at pH 5.5. Experimental NMR chemical shift perturbations (blue spheres) are mapped onto GMCSF, and NMR resonances in the presence (red) and absence (black) of heparin are shown to correlate with the proposed binding site. D) An overlay of GMCSF structures at pH 7.4 (blue, PDB: 2GMF) and pH 5.5 (red, from simulations), where disruption of $\mathrm{H} 15, \mathrm{H} 83$ and $\mathrm{H} 87$ pistacking alignment is clear at acidic $\mathrm{pH}$ and in the presence of heparin.

have modulated the same enzymatic and CD74 activities from different locations within MIF, (Cournia et al., 2009; Cho et al., 2010; Trivedi-Parmar and Jorgensen, 2018), presenting a new avenue for structure-function evaluations.

\section{CASE STUDY II: GRANULOCYTE MACROPHAGE COLONY-STIMULATING FACTOR}

Granulocyte macrophage colony-stimulating factor (GMCSF) is involved in myelopoiesis (Becher et al., 2016; Ushach and Zlotnik, 2016), immunomodulation (Lotfi et al., 2019), and proinflammatory signaling (Bhattacharya et al., 2015; Hamilton, 2019), specifically the promotion of alveolar macrophage activity leading to surfactant accumulation in the lungs (Antoniu, 2010; Kumar et al., 2018). GMCSF has been shown to aggravate conditions such as rheumatoid arthritis and multiple sclerosis (van Nieuwenhuijze et al., 2013; Shiomi et al., 2016), but it can also ameliorate diseases such as type-I diabetes (Frydrych et al., 2019), and is used clinically to combat neutropenia (Mehta et al., 2015). As such, it is a clear example of a cytokine that performs both beneficial and pathological functions. Interestingly, several studies have identified alternate receptor conformations of stoichiometric assembly that can lead to receptor activation (i.e. cell survival, activation, or differentiation) (Hansen et al., 2008; Broughton et al., 2016) and beyond its receptor structure, GMCSF itself has been shown to adopt different structural and dynamic properties dependent on the $\mathrm{pH}$ of its chemical environment, which may contribute to its modular signaling or suggest additional nonoverlapping roles for GMCSF (Cui et al., 2020).

The GMCSF structure has been studied by X-ray crystallography (Rozwarski et al., 1996), and more recently 
NMR spectroscopy (Aubin et al., 2008), providing unique insight into its overall fold and plasticity. One particular aspect of GMCSF function that has benefitted substantially from atomic level studies has been the knowledge of its interactions with clinically relevant molecules. In these cases, there has been particular interest in understanding how GMCSF, a clinically administered protein with a promiscuous interactome, could interfere with other therapeutics under conditions of disease. For example, Wettreich and coworkers showed GMCSF to form $\mathrm{pH}$-dependent complexes with heparin, another widely used therapeutic, via analytical chromatography and light scattering (Wettreich et al., 1999). These experiments proposed a binding mechanism and laid the groundwork for understanding how signaling proteins like GMCSF can be tuned by environmental stimuli to form selective ligand complexes. The GMCSF-heparin interaction has since been resolved at the atomic level with NMR spectroscopy under varied solution $\mathrm{pH}$ conditions and in the presence of precisely controlled heparin oligosaccharide chain lengths (Cui et al., 2020). The GMCSF protein was found to undergo $\mathrm{pH}$ dependent structural and dynamic changes that modulated its affinity for heparin. Such a $\mathrm{pH}$ shift would occur at airlung interfaces or in tumor microenvironments and was proposed to be essential for the ionization of three histidine residues that nucleate the heparin and the stimulation of multi-timescale dynamics that shift the conformation of $\mathrm{N}$-terminal $a$-helices to expose the binding pocket. NMR chemical shifts mapped the heparin binding site and relaxation experiments, supported by molecular simulations, confirmed a high level of conformational flexibility along the binding interface and proposed a model for the complex structure (Figure 1C). This binding interaction is dependent not only on the accessibility of the binding pocket, but the size of the heparin as well, where heparins able to span the anchor points at the histidine triad (Figure 1D) and the adjacent flexible linker with several lysine residues bind with greater affinity than smaller chain heparins.

Atomic level studies of GMCSF mapped a clinically-relevant interaction to reveal the binding site and the biophysical factors that influence its accessibility to ligands. This work also reinforced an important aspect of cytokine biochemistry that is intimately tied to the hypothesized dynamic mechanisms of action; the utility of conducting biophysical studies in regimes beyond those of "ideal" conditions. In this case, if GMCSF were only characterized based on its presence in a certain niche (i.e. a microenvironment at neutral $\mathrm{pH}$ mimicking only circulating GMCSF), the resulting biochemical outcomes would not have captured its high affinity state for heparin and a gap in our understanding of its mechanism(s) would remain. Put another way, if GMCSF is expressed in high quantities in acidic environments and in the presence of high heparin concentrations, a measurable amount of biological activity presumed to be related to the GMCSF concentration would likely not correlate with its expected activity-toquantity ratio.

\section{CASE STUDY III: INTERLEUKIN-1}

Interleukins are a diverse class of cytokines with low sequence identity that are secreted by several cell types (beyond the originally presumed leukocyte-specific secretion that spawned their name). One subclass, the interleukin (IL)-1 family, comprises 11 pro- and anti-inflammatory cytokines that have abnormal expression profiles in many autoimmune diseases (Dinarello, 2018), which presumably affects their signal transduction by the IL-1 receptor (IL-1R). Interestingly, Ge et al recently demonstrated that IL- $1 \mathrm{R}$ regulates its signaling activity via structural dynamics (Ge et al., 2019). Most IL-1 family members are commonly expressed as full-length precursors that require proteolytic processing for biologically mature forms, leading to significant structural variability. IL-1 $\alpha$ is cleaved by the cysteine protease calpain, whereas IL- $1 \beta$ and IL-18 require proteolytic cleavage by the inflammasome (Black et al., 1988; van de Veerdonk et al., 2011). IL-33 and IL-36 utilize neutrophil proteinases such as elastase and proteinase-3 (Clancy et al., 2018), while IL-37 is cleaved by capsase-1 (Kumar et al., 2002) and IL-38 is bioactive as a full-length molecule. Mechanisms between members of the IL-1 family differ in binding interactions with the receptor machinery as Günther et al. have shown that IL-1 $\beta$ and IL-33 use different molecular mechanisms for engaging with the IL-1 receptor accessory protein (IL-1Ra) (Günther et al., 2017). While Hou et al. took an alternate approach by leveraging different binding characteristics of IL- $1 \beta$ and IL-1Ra to engineer chimeric receptor antagonists of IL-1R to alleviate the chronically inflammatory dry eye disease (Hou et al., 2013).

Several groups have explored the biophysical aspects of these structures in order to understand how IL-1s transduce signals throughout the cell. One example is the computational approach taken by Ozbabakan et al to map the structural postures of IL-1, IL-1R1 and IL-1RAP as well as downstream signaling proteins (such as MYD88 and TOLLIP) when previously identified oncogenic SNPs cause changes in amino acid residues (Acuner Ozbabacan et al., 2014). This study highlights the change in protein dynamics and its subsequent effect on an entire downstream signaling pathway. The authors present a computationally derived structural pathway detailing the conformational changes related to IL-1 signaling, revealing the mechanisms behind mutations that lead to oncogenic consequences. This work is one example that underscores the importance of atomistic shifts in cytokine structure (rather than a global ensemble) as valuable in the pursuit of targeted therapies and explorations of regulatory mechanisms.

\section{CASE STUDY IV: INTERLEUKIN-2}

IL-2 is produced predominantly by activated T-cells in secondary lymphoid organs, where it is consumed by these and other CD $25^{+}$ cells, including regulatory $\mathrm{T}$-cells $\left(\mathrm{T}_{\mathrm{Reg}}\right)$ and lymphocytes. IL-2 has been used in both agonistic and antagonistic contexts for immunotherapies, including 1) low-dose efforts to increase $T_{\text {Reg }}$ cell counts in autoimmunity, chronic inflammatory conditions and graft rejection (Tahvildari and Dana, 2019) and 2) high-dose 
A

B

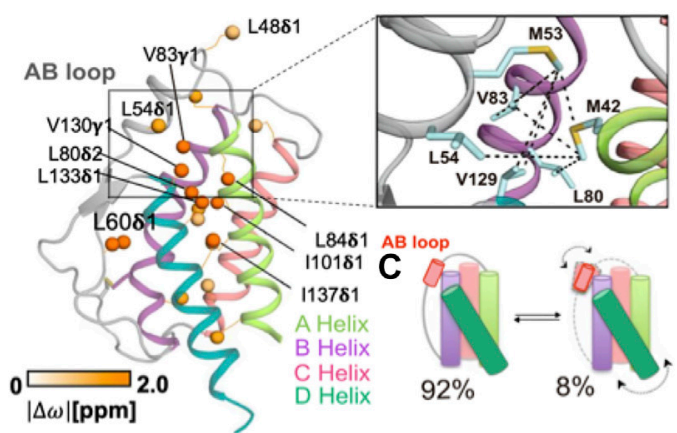

D

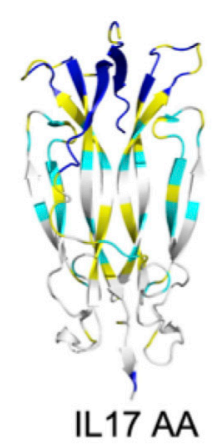

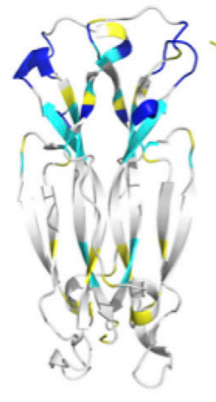

IL17 FF

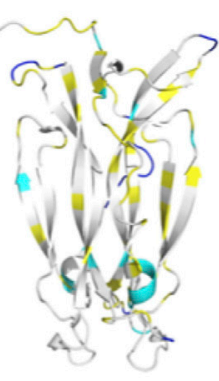

IL17 AF

FIGURE 2 | Molecular insights from structure-function studies of interleukins. (A) Methyl side chains probed at carbons via C (Conlon et al., 2019) labeling in the core of IL-2 undergoing chemical exchange are shown as orange spheres. (B) Close-up view of the AB loop showing a network (dotted lines) of observed NOEs in the IL2 ground state, where the AB loop is well-packed against the hydrophobic core. (C) Schematic representation of IL-2 interconversion between ground state (92\% population) and excited state (8\% populated). Arrows represent concerted conformational changes in the AB loop and hydrophobic core. Figures $\mathbf{2 A - C}$ is reproduced with small orientational modifications done in Procreate and PowerPoint from De Paula et al. (2020). (D) Cartoon representations of IL-17 in dimeric states, highlighting altered regions of dynamics. ${ }^{15} \mathrm{~N}-{ }^{1} \mathrm{H}$ HSQC NMR spectra collected of each IL-17 dimer were compared and regions with differences in backbone amide peak intensities were mapped onto the structure. Residues highlighted in cyan display significantly decreased peak intensities or give rise to double resonances, suggesting highly dynamic sites. Residues highlighted in yellow are amino acids for which peak intensity could not be determined based on spectral overlap, while residues highlighted in blue are unassigned. Figure 2D is reprinted from Cytokine vol. 142, Waters et al., "Conformational dynamics in interleukin 17A and 17F functional complexes is a key determinant of receptor A affinity and specificity." 2021. With permission from Elsevier.

administration to expand cytotoxic lymphocyte populations for the treatment of metastatic cancer (Jiang et al., 2016; Buchbinder et al., 2019). Efforts to understand the signaling mechanism and therapeutic potential of IL-2 have focused primarily on cellular and live animal studies, though others have tackled questions relating to its molecular structure and conformational landscape.

Critically, it has been established that IL-2 adopts discrete structures in solution that are populated via allosteric activation as shown by Sgourakis and coworkers (De Paula et al., 2020). These results build upon a previous body of literature that clearly demonstrate divergent cell fates based on signaling related to the structural plasticity of IL-2. Specifically, methyl-based chemical exchange NMR spectroscopy interrogated IL-2 dynamics on a timescale $(\mu s-m s)$ relevant to enzyme catalysis, ligand binding, and allostery (Figures 2A,B) (Lisi and Loria, 2016). The authors reported that a flexible loop between two N-terminal $a$-helices acts as a switch (Figure 2C), toggling between an autoinhibited state that has greater affinity for antibody binding, leading to proliferation of $\mathrm{T}_{\text {reg }}$ cell populations, and a productive state, where IL-2 is primed to interact with IL-2 receptor- $\alpha$, leading to activation of $\mathrm{T}_{\text {eff }}$ (effector) cell populations (De Paula et al., 2020). De Paula et al. go on to propose an allosteric network of residues serving as the link between the hydrophobic core of IL-2 and the $\mathrm{AB}$ loop (Figure 2B), highlighting how a focus on localized molecular motions can lead to novel and detailed mechanistic insight about a cytokine that can be both leveraged and accounted for in future therapeutic efforts.

\section{CASE STUDY V: INTERLEUKIN-17}

IL-17 promotes inflammation and plays a protective role in antimicrobial immunity (McGeachy et al., 2019). In the latter case, IL-17 was shown to mediate protection against extracellular pathogens (Valeri and Raffatellu, 2016; Eyerich et al., 2017) by working with IL-22 (a related cytokine also produced by IL17-expressing cells) to stimulate production of antimicrobial peptides (Wozniak et al., 2014; Mulcahy et al., 2016; Moyat et al., 2017). IL-17 is therefore a double-edged sword with biological properties that make it difficult to predict its role in inflammatory diseases with a polymicrobial etiology. It is possible that IL-17 exerts both protective and destructive effects, as suggested in distinct mouse models (Borkner et al., 2021; Milovanovic et al., 2020; Cruz et al., 2010; Dallenbach et al., 2015; Righetti et al., 2018; O'Connor Jr et al., 2009), although chronic IL-17 receptor signaling can turn a potentially protective acute inflammatory response into chronic immunopathology (Zenobia and Hajishengallis, 2015).

Despite its status as one of best studied cytokines in immunology, recent structure-function relationships and dynamic studies have provided new insight into its regulatory mechanism. Specifically, IL-17 conformational dynamics were shown to dictate signaling efficiency via a strong influence over binding affinity for this system. The structural interconversions of IL-17A and IL-17F were studied with NMR spectroscopy by Waters et al, where the IL-17A homodimer was demonstrated to be far more dynamic on slow timescales than the IL-17F homodimer or IL-17A/IL-17F heterodimer (Figure 2D). The aforementioned dynamics aid the IL-17A homodimer in toggling between at least two distinct conformations and the structural perturbations during this process were mapped to the receptor binding region (Waters et al., 2021). The authors propose that these structural fluctuations are likely to affect binding affinity to receptor A (but not other IL-17 receptors), providing insight into the activation of receptor signaling based on atomic level dynamics that cannot be resolved from systems 
level observations examining only the presence and quantity of IL-17.

\section{CONCLUDING REMARKS}

The mechanisms by which cytokines influence a large number of biochemical pathways and molecular interactions have been mysterious. The fact that most proteins in this class have very small structures with simple folds only adds to this paradox. It is difficult to link all of cytokine biochemistry with a common thread, primarily because the majority of information regarding cytokine functions comes from cellular or live animal studies, with comparatively few biophysical investigations. However, the current literature describing cytokine structure has generally shown that 1) protein dynamics that toggle active conformations of cytokines are a driver of functional

\section{REFERENCES}

Acuner Ozbabacan, S. E., Gursoy, A., Nussinov, R., and Keskin, O. (2014). The Structural Pathway of Interleukin 1 (IL-1) Initiated Signaling Reveals Mechanisms of Oncogenic Mutations and SNPs in Inflammation and Cancer. Plos Comput. Biol. 10. e1003470 doi:10.1371/journal.pcbi.1003470

Altan-Bonnet, G., and Mukherjee, R. (2019). Cytokine-mediated Communication: a Quantitative Appraisal of Immune Complexity. Nat. Rev. Immunol. 19, 205-217. doi:10.1038/s41577-019-0131-x

Antoniu, S. A. (2010). GM-CSF Pathway Correction in Pulmonary Alveolar Proteinosis. Expert Opin. Biol. Ther. 10, 1357-1365. doi:10.1517/ 14712598.2010 .510507

Arango Duque, G., and Descoteaux, A. (2014). Macrophage Cytokines: Involvement in Immunity and Infectious Diseases. Front. Immunol. 5, 491. doi:10.3389/fimmu.2014.00491

Aubin, Y., Gingras, G., and Sauvé, S. (2008). Assessment of the Three-Dimensional Structure of Recombinant Protein Therapeutics by NMR Fingerprinting: Demonstration on Recombinant Human Granulocyte Macrophage-colony Stimulation Factor. Anal. Chem. 80, 2623-2627. doi:10.1021/ac7026222

Azimzadeh Irani, M., and Ejtehadi, M. R. (2019). GAG Positioning on IL-1RI; A Mechanism Regulated by Dual Effect of Glycosylation. Glycobiology 29, 803-812. doi:10.1093/glycob/cwz055

Bach, J.-P., Rinn, B., Meyer, B., Dodel, R., and Bacher, M. (2008). Role of MIF in Inflammation and Tumorigenesis. Oncology 75, 127-133. doi:10.1159/000155223

Baeza Garcia, A., Siu, E., Sun, T., Exler, V., Brito, L., Hekele, A., et al. (2018). Neutralization of the Plasmodium-Encoded MIF Ortholog Confers Protective Immunity against Malaria Infection. Nat. Commun. 9, 2714. doi:10.1038/ s41467-018-05041-7

Barnes, B. J., and Somerville, C. C. (2020). Modulating Cytokine Production via Select Packaging and Secretion from Extracellular Vesicles. Front. Immunol. 11, 1040. doi:10.3389/fimmu.2020.01040

Becher, B., Tugues, S., and Greter, M. (2016). GM-CSF: From Growth Factor to Central Mediator of Tissue Inflammation. Immunity 45, 963-973. doi:10.1016/ j.immuni.2016.10.026

Bendrat, K., Al-Abed, Y., Callaway, D. J. E., Peng, T., Calandra, T., Metz, C. N., et al. (1997). Biochemical and Mutational Investigations of the Enzymatic Activity of Macrophage Migration Inhibitory Factor. Biochemistry 36, 15356-15362. doi:10.1021/bi971153a

Berghaus, L. J., Giguère, S., Bordin, A. I., and Cohen, N. D. (2018). Effects of Priming with Cytokines on Intracellular Survival and Replication of Rhodococcus Equi in Equine Macrophages. Cytokine 102, 7-11. doi:10.1016/ j.cyto.2017.12.011

Berraondo, P., Sanmamed, M. F., Ochoa, M. C., Etxeberria, I., Aznar, M. A., PérezGracia, J. L., et al. (2019). Cytokines in Clinical Cancer Immunotherapy. Br. J. Cancer 120, 6-15. doi:10.1038/s41416-018-0328-y promiscuity, 2) the cellular or chemical environment modulates cytokine structure and interactions with specific binding partners, and 3) despite their small size, mechanisms of cytokine regulation can be driven by principles of allostery and concerted motion.

\section{AUTHOR CONTRIBUTIONS}

All authors listed have made a substantial, direct, and intellectual contribution to the work and approved it for publication.

\section{FUNDING}

This work was supported by Rhode Island Foundation Grant GR5290658.

Bhattacharya, P., Budnick, I., Singh, M., Thiruppathi, M., Alharshawi, K., Elshabrawy, H., et al. (2015). Dual Role of GM-CSF as a Pro-inflammatory and a Regulatory Cytokine: Implications for Immune Therapy. J. Interferon Cytokine Res. 35, 585-599. doi:10.1089/jir.2014.0149

Black, R. A., Kronheim, S. R. K., Cantrell, M., Deeley, M. C., March, C. J., Prickett, K. S., et al. (1988). Generation of Biologically Active Interleukin-1 Beta by Proteolytic Cleavage of the Inactive Precursor. J. Biol. Chem. 263, 5. doi:10.1016/s0021-9258(19)76559-4

Borkner, L., Curham, L. M., Wilk, M. M., Moran, B., and Mills, K. H. G. (2021). IL17 Mediates Protective Immunity against Nasal Infection with Bordetella Pertussis by Mobilizing Neutrophils, Especially Siglec-F+ Neutrophils. Mucosal Immunol. 14, 1183-1202. doi:10.1038/s41385-021-00407-5

Boukouris, A. E., Zervopoulos, S. D., and Michelakis, E. D. (2016). Metabolic Enzymes Moonlighting in the Nucleus: Metabolic Regulation of Gene Transcription. Trends in Biochemical Sciences 41, 712-730. doi:10.1016/ j.tibs.2016.05.013

Broughton, S. E., Hercus, T. R., Nero, T. L., Dottore, M., McClure, B. J., Dhagat, U., Taing, H., Gorman, M. A., King-Scott, J., Lopez, A. F., and Parker, M. W. (2016). Conformational Changes in the GM-CSF Receptor Suggest a Molecular Mechanism for Affinity Conversion and Receptor Signaling. Structure 24, 1271-1281. doi:10.1016/j.str.2016.05.017

Buchbinder, E. I., Dutcher, J. P., Daniels, G. A., Curti, B. D., Patel, S. P., Holtan, S. G., Miletello, G. P., Fishman, M. N., Gonzalez, R., Clark, J. I., Richart, J. M., Lao, C. D., Tykodi, S. S., Silk, A. W., and McDermott, D. F. (2019). Therapy with high-dose Interleukin-2 (HD IL-2) in metastatic melanoma and renal cell carcinoma following PD1 or PDL1 inhibition. j. immunotherapy cancer 7, 49. doi:10.1186/s40425-019-0522-3

Chen, C., Liu, H., Zabad, S., Rivera, N., Rowin, E., Hassan, M., Gomez De Jesus, S. M., Llinás Santos, P. S., Kravchenko, K., Mikhova, M., Ketterer, S., Shen, A., Shen, S., Navas, E., Horan, B., Raudsepp, J., and Jeffery, C. (2021). MoonProt 3.0: an update of the moonlighting proteins database. Nucleic Acids Res 49, D368-D372. doi:10.1093/nar/gkaa1101

Chen, C., Zabad, S., Liu, H., Wang, W., and Jeffery, C. (2018). MoonProt 2.0: an Expansion and Update of the Moonlighting Proteins Database. Nucleic Acids Res. 46, D640-D644. doi:10.1093/nar/gkx1043

Cho, Y., Crichlow, G. V., Vermeire, J. J., Leng, L., Du, X., Hodsdon, M. E., et al. (2010). Allosteric Inhibition of Macrophage Migration Inhibitory Factor Revealed by Ibudilast. Proc. Natl. Acad. Sci. 107, 11313-11318. doi:10.1073/ pnas. 1002716107

Clancy, D. M., Sullivan, G. P., Moran, H. B. T., Henry, C. M., Reeves, E. P., McElvaney, N. G., et al. (2018). Extracellular Neutrophil Proteases Are Efficient Regulators of IL-1, IL-33, and IL-36 Cytokine Activity but Poor Effectors of Microbial Killing. Cel Rep. 22, 2937-2950. doi:10.1016/j.celrep.2018.02.062

Conlon, K. C., Miljkovic, M. D., and Waldmann, T. A. (2019). Cytokines in the Treatment of Cancer. J. Interferon Cytokine Res. 39, 6-21. doi:10.1089/ jir.2018.0019 
Cournia, Z., Leng, L., Gandavadi, S., Du, X., Bucala, R., and Jorgensen, W. L. (2009). Discovery of Human Macrophage Migration Inhibitory Factor (MIF)-CD74 Antagonists via Virtual Screening. J. Med. Chem. 52, 416-424. doi:10.1021/ jm801100v

Cruz, A., Fraga, A. G., Fountain, J. J., Rangel-Moreno, J., Torrado, E., Saraiva, M., et al. (2010). Pathological Role of Interleukin 17 in Mice Subjected to Repeated BCG Vaccination after Infection with Mycobacterium tuberculosis. J. Exp. Med. 207, 1609-1616. doi:10.1084/jem.20100265

Cui, J. Y., Zhang, F., Nierzwicki, L., Palermo, G., Linhardt, R. J., and Lisi, G. P. (2020). Mapping the Structural and Dynamic Determinants of $\mathrm{pH}$-Sensitive Heparin Binding to Granulocyte Macrophage Colony Stimulating Factor. Biochemistry 59, 3541-3553. doi:10.1021/acs.biochem.0c00538

Dallenbach, K., Maurer, P., Röhn, T., Zabel, F., Kopf, M., and Bachmann, M. F. (2015). Protective Effect of a Germline, IL-17-neutralizing Antibody in Murine Models of Autoimmune Inflammatory Disease. Eur. J. Immunol. 45, 1238-1247. doi:10.1002/eji.201445017

de Jong, Y. P., Abadia-Molina, A. C., Satoskar, A. R., Clarke, K., Rietdijk, S. T., Faubion, W. A., et al. (2001). Development of Chronic Colitis Is Dependent on the Cytokine MIF. Nat. Immunol. 2, 1061-1066. doi:10.1038/ni720

De Paula, V. S., Jude, K. M., Nerli, S., Glassman, C. R., Garcia, K. C., and Sgourakis, N. G. (2020). Interleukin-2 Druggability Is Modulated by Global Conformational Transitions Controlled by a Helical Capping Switch. Proc. Natl. Acad. Sci. USA 117, 7183-7192. doi:10.1073/pnas.2000419117

Dinarello, C. A. (2018). Overview of the IL-1 Family in Innate Inflammation and Acquired Immunity. Immunol. Rev. 281, 8-27. doi:10.1111/imr.12621

Donnelly, S. C., Haslett, C., Reid, P. T., Grant, I. S., Wallace, W. A. H., Metz, C. N., et al. (1997). Regulatory Role for Macrophage Migration Inhibitory Factor in Acute Respiratory Distress Syndrome. Nat. Med. 3, 320-323. doi:10.1038/ nm0397-320

El-Turk, F., Cascella, M., Ouertatani-Sakouhi, H., Narayanan, R. L., Leng, L., Bucala, R., et al. (2008). The Conformational Flexibility of the Carboxy Terminal Residues 105-114 Is a Key Modulator of the Catalytic Activity and Stability of Macrophage Migration Inhibitory Factor $\dagger$. Biochemistry 47, 10740-10756. doi:10.1021/bi800603x

El-Turk, F., Fauvet, B., Ashrafi, A., Ouertatani-Sakouhi, H., Cho, M.-K., Neri, M., et al. (2012). Characterization of Molecular Determinants of the Conformational Stability of Macrophage Migration Inhibitory Factor: Leucine 46 Hydrophobic Pocket. PLoS One 7. e45024 doi:10.1371/ journal.pone.0045024

Eyerich, K., Dimartino, V., and Cavani, A. (2017). IL-17 and IL-22 in Immunity: Driving protection and Pathology. Eur. J. Immunol. 47, 607-614. doi:10.1002/ eji.201646723

Fan, C., Rajasekaran, D., Syed, M. A., Leng, L., Loria, J. P., Bhandari, V., et al. (2013). MIF Intersubunit Disulfide Mutant Antagonist Supports Activation of CD74 by Endogenous MIF Trimer at Physiologic Concentrations. Proc. Natl. Acad. Sci. 110, 10994-10999. doi:10.1073/pnas.1221817110

Frydrych, L. M., Bian, G., Fattahi, F., Morris, S. B., O’Rourke, R. W., Lumeng, C. N., et al. (2019). GM-CSF Administration Improves Defects in Innate Immunity and Sepsis Survival in Obese Diabetic Mice. J.I. 202, 931-942. doi:10.4049/jimmunol.1800713

Gangele, K., Gulati, K., Joshi, N., Kumar, D., and Poluri, K. M. (2020). Molecular Insights into the Differential Structure-Dynamics-Stability Features of Interleukin-8 Orthologs: Implications to Functional Specificity. Int. J. Biol. Macromolecules 164, 3221-3234. doi:10.1016/j.ijbiomac.2020.08.176

Ge, J., Remesh, S. G., Hammel, M., Pan, S., Mahan, A. D., Wang, S., et al. (2019). Functional Relevance of Interleukin-1 Receptor Inter-domain Flexibility for Cytokine Binding and Signaling. Structure 27, 1296-1307. e1295 doi:10.1016/ j.str.2019.05.011

Günther, S., Deredge, D., Bowers, A. L., Luchini, A., Bonsor, D. A., Beadenkopf, R., et al. (2017). IL-1 Family Cytokines Use Distinct Molecular Mechanisms to Signal through Their Shared Co-receptor. Immunity 47, 510-523. e514 doi:10.1016/j.immuni.2017.08.004

Hamilton, J. A. (2019). GM-CSF-Dependent Inflammatory Pathways. Front. Immunol. 10, 2055. doi:10.3389/fimmu.2019.02055

Han, C., Lin, Y., Shan, G., Zhang, Z., Sun, X., Wang, Z., et al. (2010). Plasma Concentration of Malaria Parasite-Derived Macrophage Migration Inhibitory Factor in Uncomplicated Malaria Patients Correlates with Parasitemia and Disease Severity. Clin. Vaccin. Immunol 17, 1524-1532. doi:10.1128/ CVI.00149-10
Hansen, G., Hercus, T. R., McClure, B. J., Stomski, F. C., Dottore, M., Powell, J., et al. (2008). The Structure of the GM-CSF Receptor Complex Reveals a Distinct Mode of Cytokine Receptor Activation. Cell 134, 496-507. doi:10.1016/j.cell.2008.05.053

Herring, C. A., Singer, C. M., Ermakova, E. A., Khairutdinov, B. I., Zuev, Y. F., Jacobs, D. J., et al. (2015). Dynamics and Thermodynamic Properties of CXCL7 Chemokine. Proteins 83, 1987-2007. doi:10.1002/prot.24913

Hou, J., Townson, S. A., Kovalchin, J. T., Masci, A., Kiner, O., Shu, Y., et al. (2013). Design of a superior Cytokine Antagonist for Topical Ophthalmic Use. Proc. Natl. Acad. Sci. 110, 3913-3918. doi:10.1073/ pnas. 1217996110

Hudson, J. D., Shoaibi, M. A., Maestro, R., Carnero, A., Hannon, G. J., and Beach, D. H. (1999). A Proinflammatory Cytokine Inhibits P53 Tumor Suppressor Activity. J. Exp. Med. 190, 1375-1382. doi:10.1084/ jem.190.10.1375

Jiang, T., Zhou, C., and Ren, S. (2016). Role of IL-2 in Cancer Immunotherapy. Oncoimmunology 5. e1163462 doi:10.1080/2162402X.2016.1163462

Joseph, P. R. B., Mosier, P. D., Desai, U. R., and Rajarathnam, K. (2015). Solution NMR Characterization of Chemokine CXCL8/IL-8 Monomer and Dimer Binding to Glycosaminoglycans: Structural Plasticity Mediates Differential Binding Interactions. Biochem. J. 472, 121-133. doi:10.1042/BJ20150059

Kämpfer, A. A. M., Urbán, P., Gioria, S., Kanase, N., Stone, V., and KinsnerOvaskainen, A. (2017). Development of an In Vitro Co-culture Model to Mimic the Human Intestine in Healthy and Diseased State. Toxicol. Vitro 45, 31-43. doi:10.1016/j.tiv.2017.08.011

Kim, H. R. P. M., Park, M. K., Cho, M. L., Yoon, C. H., Lee, S. H., Park, S. H., et al. (2007). Macrophage Migration Inhibitory Factor Upregulates Angiogenic Factors and Correlates with Clinical Measures in Rheumatoid Arthritis. J. Rheumatol. 34, 927-936.

Kleemann, R., Kapurniotu, A., Frank, R. W., Gessner, A., Mischke, R., Flieger, O., et al. (1998). Disulfide Analysis Reveals a Role for Macrophage Migration Inhibitory Factor (MIF) as Thiol-Protein Oxidoreductase. J. Mol. Biol. 280, 85-102. doi:10.1006/jmbi.1998.1864

Kumar, A., Abdelmalak, B., Inoue, Y., and Culver, D. A. (2018). Pulmonary Alveolar Proteinosis in Adults: Pathophysiology and Clinical Approach. Lancet Respir. Med. 6, 554-565. doi:10.1016/s2213-2600(18)30043-2

Kumar, S., Hanning, C. R., Brigham-Burke, M. R., Rieman, D. J., Lehr, R., Khandekar, S., et al. (2002). Interleukin-1f7b (Il-1h4/Il-1f7) Is Processed By Caspase-1 And Mature Il-1f7b Binds To The Il-18 Receptor But Does Not Induce Ifn- $\Gamma$ Production. Cytokine 18, 61-71. doi:10.1006/cyto.2002.0873

Künze, G., Gehrcke, J.-P., Pisabarro, M. T., and Huster, D. (2014). NMR Characterization of the Binding Properties and Conformation of Glycosaminoglycans Interacting with Interleukin-10. Glycobiology 24, 1036-1049. doi:10.1093/glycob/cwu069

Künze, G., Köhling, S., Vogel, A., Rademann, J., and Huster, D. (2016). Identification of the Glycosaminoglycan Binding Site of Interleukin-10 by NMR Spectroscopy. J. Biol. Chem. 291, 3100-3113. doi:10.1074/jbc.M115.681759

Lai, K. N., Leung, J. C., Metz, C. N., Lai, F. M., Bucala, R., and Lan, H. Y. (2003). Role for Macrophage Migration Inhibitory Factor in Acute Respiratory Distress Syndrome. J. Pathol. 199, 496-508. doi:10.1002/path.1291

Lisi, G. P., and Loria, J. P. (2016). Solution NMR Spectroscopy for the Study of Enzyme Allostery. Chem. Rev. 116, 6323-6369. doi:10.1021/ acs.chemrev.5b00541

Llamas-Covarrubias, M. A., Valle, Y., Navarro-Hernández, R. E., GuzmánGuzmán, I. P., Ramírez-Dueñas, M. G., Rangel-Villalobos, H., et al. (2012). Serum Levels of Macrophage Migration Inhibitory Factor Are Associated with Rheumatoid Arthritis Course. Rheumatol. Int. 32, 2307-2311. doi:10.1007/ s00296-011-1951-6

Lotfi, N., Thome, R., Rezaei, N., Zhang, G.-X., Rezaei, A., Rostami, A., et al. (2019). Roles of GM-CSF in the Pathogenesis of Autoimmune Diseases: An Update. Front. Immunol. 10, 1265. doi:10.3389/fimmu.2019.01265

Lubetsky, J. B., Swope, M., Dealwis, C., Blake, P., and Lolis, E. (1999). Pro-1 of Macrophage Migration Inhibitory Factor Functions as a Catalytic Base in the Phenylpyruvate Tautomerase Activity,. Biochemistry 38, 7346-7354. doi:10.1021/bi990306m

Mani, M., Chen, C., Amblee, V., Liu, H., Mathur, T., Zwicke, G., et al. (2015). MoonProt: a Database for Proteins that Are Known to Moonlight. Nucleic Acids Res. 43, D277-D282. doi:10.1093/nar/gku954 
McGeachy, M. J., Cua, D. J., and Gaffen, S. L. (2019). The IL-17 Family of Cytokines in Health and Disease. Immunity 50, 892-906. doi:10.1016/j.immuni.2019.03.021

Mehta, H. M., Malandra, M., and Corey, S. J. (2015). G-CSF and GM-CSF in Neutropenia. J. Immunol. 195, 1341-1349. doi:10.4049/ jimmunol.1500861

Milovanovic, J., Arsenijevic, A., Stojanovic, B., Kanjevac, T., Arsenijevic, D., Radosavljevic, G., et al. (2020). Interleukin-17 in Chronic Inflammatory Neurological Diseases. Front. Immunol. 11, 947. doi:10.3389/ fimmu. 2020.00947

Mitchell, R. A., Liao, H., Chesney, J., Fingerle-Rowson, G., Baugh, J., David, J., et al. (2002). Macrophage Migration Inhibitory Factor (MIF) Sustains Macrophage Proinflammatory Function by Inhibiting P53: Regulatory Role in the Innate Immune Response. Proc. Natl. Acad. Sci. 99, 345-350. doi:10.1073/ pnas. 012511599

Moyat, M., Bouzourene, H., Ouyang, W., Iovanna, J., Renauld, J.-C., and Velin, D. (2017). IL-22-induced Antimicrobial Peptides Are Key Determinants of Mucosal Vaccine-Induced protection against $H$. pylori in Mice. Mucosal Immunol. 10, 271-281. doi:10.1038/mi.2016.38

Mühlhahn, P., Czisch, M., Georgescu, J., Renner, C., Ross, A., Holak, T. A., et al. (1996). NMR Characterization of Structure, Backbone Dynamics, and Glutathione Binding of the Human Macrophage Migration Inhibitory Factor (MIF). Protein Sci. 5, 2095-2103. doi:10.1002/ pro.5560051016

Mulcahy, M. E., Leech, J. M., Renauld, J.-C., Mills, K. H., and McLoughlin, R. M. (2016). Interleukin-22 Regulates Antimicrobial Peptide Expression and Keratinocyte Differentiation to Control Staphylococcus aureus Colonization of the Nasal Mucosa. Mucosal Immunol. 9, 1429-1441. doi: $10.1038 / \mathrm{mi} .2016 .24$

Noe, J. T., and Mitchell, R. A. (2020). MIF-dependent Control of Tumor Immunity. Front. Immunol. 11. 609948 doi:10.3389/fimmu.2020.609948

O'Connor Jr, W., Jr., Kamanaka, M., Booth, C. J., Town, T., Nakae, S., Iwakura, Y., et al. (2009). A Protective Function for Interleukin 17A in T Cell-Mediated Intestinal Inflammation. Nat. Immunol. 10, 603-609. doi:10.1038/ni.1736

Oda, S., Oda, T., Nishi, K., Takabuchi, S., Wakamatsu, T., Tanaka, T., et al. (2008). Macrophage Migration Inhibitory Factor Activates Hypoxia-Inducible Factor in a P53-dependent Manner. PLoS One 3, e2215. doi:10.1371/ journal.pone. 0002215

Onodera, S., Tanji, H., Suzuki, K., Kaneda, K., Mizue, Y., Sagawa, A., et al. (1999). High Expression of Macrophage Migration Inhibitory Factor in the Synovial Tissues of Rheumatoid Joints. Cytokine 11, 163-167. doi:10.1006/ cyto.1998.0402

Pantouris, G., Ho, J., Shah, D., Syed, M. A., Leng, L., Bhandari, V., et al. (2018). Nanosecond Dynamics Regulate the MIF-Induced Activity of CD74. Angew. Chem. Int. Ed. 57, 7116-7119. doi:10.1002/ anie.201803191

Pantouris, G., Khurana, L., Ma, A., Skeens, E., Reiss, K., Batista, V. S., et al. (2020). Regulation of MIF Enzymatic Activity by an Allosteric Site at the Central Solvent Channel. Cel Chem. Biol. 27, 740-750. e745 doi:10.1016/ j.chembiol.2020.05.001

Pantouris, G., Syed, M. A., Fan, C., Rajasekaran, D., Cho, T. Y., Rosenberg, E. M., et al. (2015). An Analysis of MIF Structural Features that Control Functional Activation of CD74. Chem. Biol. 22, 1197-1205. doi:10.1016/ j.chembiol.2015.08.006

Park, S. H., Berkamp, S., Radoicic, J., De Angelis, A. A., and Opella, S. J. (2017). Interaction of Monomeric Interleukin-8 with CXCR1 Mapped by ProtonDetected Fast MAS Solid-State NMR. Biophysical J. 113, 2695-2705. doi:10.1016/j.bpj.2017.09.041

Parkins, A., Skeens, E., McCallum, C. M., Lisi, G. P., and Pantouris, G. (2021). The N-Terminus of MIF Regulates the Dynamic Profile of Residues Involved in CD74 Activation. Biophysical J. 120, 3893-3900. doi:10.1016/j.bpj.2021.08.025

Penk, A., Baumann, L., Huster, D., and Samsonov, S. A. (2019). NMR and Molecular Modeling Reveal Specificity of the Interactions between CXCL14 and Glycosaminoglycans. Glycobiology 29, 715-725. doi:10.1093/glycob/ cwz047

Righetti, R. F., Santos, T. M. d., Camargo, L. d. N., Aristóteles, L. R. C. R. B., Fukuzaki, S., Souza, F. C. R. d., et al. (2018). Protective Effects of Anti-IL17 on
Acute Lung Injury Induced by LPS in Mice. Front. Pharmacol. 9, 1021. doi:10.3389/fphar.2018.01021

Roan, F., Obata-Ninomiya, K., and Ziegler, S. F. (2019). Epithelial Cell-Derived Cytokines: More Than Just Signaling the Alarm. J. Clin. Invest. 129, 1441-1451. doi:10.1172/JCI124606

Roy, U. (2020). Insight into the Structures of Interleukin-18 Systems. Comput. Biol. Chem. 88, 107353. doi:10.1016/ j.compbiolchem.2020.107353

Rozwarski, D. A., Diederichs, K., Hecht, R., Boone, T., and Karplus, P. A. (1996). Refined crystal Structure and Mutagenesis of Human Granulocyte-Macrophage colony-stimulating Factor. Proteins 26, 304-313. doi:10.1002/(sici)1097-0134(199611)26:3<304:Aidprot6 $>3.0 . \mathrm{Co} ; 2-\mathrm{d}$

Schinagl, A., Kerschbaumer, R. J., Sabarth, N., Douillard, P., Scholz, P., Voelkel, D., et al. (2018). Role of the Cysteine 81 Residue of Macrophage Migration Inhibitory Factor as a Molecular Redox Switch. Biochemistry 57, 1523-1532. doi:10.1021/acs.biochem.7b01156

Schluns, K. S., and Lefrançois, L. (2003). Cytokine Control of Memory T-Cell Development and Survival. Nat. Rev. Immunol. 3, 269-279. doi:10.1038/ nri1052

Shiomi, A., Usui, T., and Mimori, T. (2016). GM-CSF as a Therapeutic Target in Autoimmune Diseases. Inflamm. Regener 36, 8. doi:10.1186/ s41232-016-0014-5

Subbannayya, T., Variar, P., Advani, J., Nair, B., Shankar, S., Gowda, H., et al. (2016). An Integrated Signal Transduction Network of Macrophage Migration Inhibitory Factor. J. Cel Commun. Signal. 10, 165-170. doi:10.1007/s12079016-0326-x

Sun, T., Holowka, T., Song, Y., Zierow, S., Leng, L., Chen, Y., et al. (2012). A Plasmodium-Encoded Cytokine Suppresses T-Cell Immunity during Malaria. Proc. Natl. Acad. Sci. 109, E2117-E2126. doi:10.1073/ pnas. 1206573109

Tahvildari, M., and Dana, R. (2019). Low-Dose IL-2 Therapy in Transplantation, Autoimmunity, and Inflammatory Diseases. J.I. 203, 2749-2755. doi:10.4049/ jimmunol.1900733

Takahashi, K., Koga, K., Linge, H. M., Zhang, Y., Lin, X., Metz, C. N., et al. (2009). Macrophage CD74 Contributes to MIF-Induced Pulmonary Inflammation. Respir. Res. 10, 33. doi:10.1186/1465-9921-10-33

Toldi, J., Nemeth, D., Hegyi, P., Molnar, Z., Solymar, M., Farkas, N., et al. (2021). Macrophage Migration Inhibitory Factor as a Diagnostic and Predictive Biomarker in Sepsis: Meta-Analysis of Clinical Trials. Sci. Rep. 11, 8051. doi:10.1038/s41598-021-87613-0

Trivedi-Parmar, V., and Jorgensen, W. L. (2018). Advances and Insights for Small Molecule Inhibition of Macrophage Migration Inhibitory Factor. J. Med. Chem. 61, 8104-8119. doi:10.1021/ acs.jmedchem.8b00589

Ushach, I., and Zlotnik, A. (2016). Biological Role of Granulocyte Macrophage colony-stimulating Factor (GM-CSF) and Macrophage colony-stimulating Factor (M-CSF) on Cells of the Myeloid Lineage. J. Leukoc. Biol. 100, 481-489. doi:10.1189/jlb.3RU0316-144R

Valeri, M., and Raffatellu, M. (2016). Cytokines IL-17 and IL-22 in the Host Response to Infection. Pathog. Dis. 74. ftw111 doi:10.1093/femspd/ ftw111

van de Veerdonk, F. L., Netea, M. G., Dinarello, C. A., and Joosten, L. A. B. (2011). Inflammasome Activation and IL-1 $\beta$ and IL-18 Processing during Infection. Trends Immunol. 32, 110-116. doi:10.1016/ j.it.2011.01.003

van Nieuwenhuijze, A., Koenders, M., Roeleveld, D., Sleeman, M. A., van den Berg, W., and Wicks, I. P. (2013). GM-CSF as a Therapeutic Target in Inflammatory Diseases. Mol. Immunol. 56, 675-682. doi:10.1016/ j.molimm.2013.05.002

Wang, Y, Chen, Y, Wang, C, Yang, M, Wang, Y, Bao, L, Wang, JE, Kim, B, Chan, KY, Xu, W, Capota, E, Ortega, J, Nijhawan, D, Li, GM, Luo, W, and Wang, Y (2021). MIF is a 3' flap nuclease that facilitates DNA replication and promotes tumor growth. Nat Commun 12, 2954. doi:10.1038/s41467021-23264-z

Waters, L. C., Veverka, V., Strong, S. L., Muskett, F. W., Dedi, N., Lawson, A. D. G., Prosser, C. E., Taylor, R. J., Henry, A. J., and Carr, M. D. (2021). Conformational dynamics in interleukin $17 \mathrm{~A}$ and $17 \mathrm{~F}$ functional complexes 
is a key determinant of receptor A affinity and specificity. Cytokine 142. 155476 doi:10.1016/j.cyto.2021.155476

Wettreich, A., Sebollela, A., Carvalho, M. A., Azevedo, S. P., Borojevic, R., Ferreira, S. T., and Coelho-Sampaio, T. (1999). Acidic pH modulates the interaction between human granulocyte-macrophage colony-stimulating factor and glycosaminoglycans. Journal of Biological Chemistry 274, 31468-31475. doi:10.1074/jbc.274.44.31468

Wozniak, K. L., Hole, C. R., Yano, J., Fidel, P. L., and Wormley, F. L. (2014). Characterization of IL-22 and antimicrobial peptide production in mice protected against pulmonary Cryptococcus neoformans infection. Microbiology (Reading) 160, 1440-1452. doi:10.1099/mic.0.073445-0

Yang, C.-Y. (2020). Comparative Analyses of the Conformational Dynamics Between the Soluble and Membrane-Bound Cytokine Receptors. Sci Rep 10, 7399. doi:10.1038/s41598-020-64034-z

Zenobia, C., and Hajishengallis, G. (2015). Basic biology and role of interleukin-17 in immunity and inflammation. Periodontol 2000 69, 142-159. doi:10.1111/prd.12083
Conflict of Interest: The authors declare that the research was conducted in the absence of any commercial or financial relationships that could be construed as a potential conflict of interest.

Publisher's Note: All claims expressed in this article are solely those of the authors and do not necessarily represent those of their affiliated organizations, or those of the publisher, the editors and the reviewers. Any product that may be evaluated in this article, or claim that may be made by its manufacturer, is not guaranteed or endorsed by the publisher.

Copyright $\odot 2021$ Cui and Lisi. This is an open-access article distributed under the terms of the Creative Commons Attribution License (CC BY). The use, distribution or reproduction in other forums is permitted, provided the original author(s) and the copyright owner(s) are credited and that the original publication in this journal is cited, in accordance with accepted academic practice. No use, distribution or reproduction is permitted which does not comply with these terms. 